Heat shock proteins (HSPs) are chaperones with highly conservative primary structure, necessary in the processes of protein folding to the most energetically advantageous conformation and maintaining their stability. HSPs perform a number of important functions in various cellular processes and are capable of modulating pathophysiological conditions at the cellular and systemic levels. An example is the high level of HSP expression in neoplastic tissues, which disrupts the apoptosis of transformed cells and promotes the processes of proliferation, invasion, and metastasis. In addition, an increasing amount of information is appearing about the participation of HSPs in the formation of multidrug resistance. This paper provides a review of the current state of research on the fundamental importance as well as the diagnostic and prognostic role of various classes of HSP in cancer treatment. It presents the prospects for using HSPs as biological markers of disease progression and targets in various cancer treatment strategies. However, the need for additional research is quite high. Only numerous joint efforts of research groups will allow the effective use of HSPs as a tool to combat cancer.

Key words: cancer, heat shock proteins, apoptosis, anti-cancer therapy.

Contemp oncol (Pozn) 2021; 25 (2): 73-79 DOI: https://doi.org/10.5114/wo.2021.106006

\section{The role of heat shock proteins in neoplastic processes and the research on their importance in the diagnosis and treatment of cancer}

\author{
Iryna Boliukh ${ }^{1}$, Agnieszka Rombel-Bryzek², Olga Żuk ${ }^{1}$, Barbara Radecka³ \\ ${ }^{1}$ Institute of Environmental Engineering and Biotechnology, University of Opole, \\ Opole, Poland \\ ${ }^{2}$ Institute of Medical Sciences, University of Opole, Opole, Poland \\ ${ }^{3}$ Department of Oncology, Institute of Medical Sciences, University of Opole, Opole, Poland \\ ${ }^{4}$ Department of Clinical Oncology, Tadeusz Koszarowski Cancer Centre in Opole, \\ Opole, Poland
}

\section{Introduction}

Heat shock proteins (HSPs) are an evolutionary old family of proteins with highly conservative primary structure (invariable regardless of organism). Their characteristic feature is the ability to interact with a large number of other proteins, which clearly distinguishes HSPs from most cellular proteins that usually interact with one or more molecular counterparts. HSPs take part in various cellular processes, such as protein folding into the most energetically advantageous conformation, transporting proteins through membranes, protecting and controlling their structure, processing antigens, or binding peptides to molecules of major histocompatibility complex class I [1, 2]. The main task of HSPs is to protect the cell by suppressing the effects of various stress factors, and therefore they are sometimes called chaperones. In a properly developing cell, HSPs constitute approx. 5-10\% of the total amount of proteins. The transcription of HSP encoding genes and the increase in expression of these proteins is triggered by various environmental stress factors (e.g. high temperature, metabolic poisons, ionizing radiation) and in the course of pathophysiological processes in the body (viral and bacterial infections, as well as cancer). Because apoptosis and HSP expression are induced by the same factors, researchers have sought a correlation between these 2 processes. An extremely important role of HSPs is the ability to model the early stages of apoptosis, i.e. programmed cell death; therefore, in the last few decades these proteins have become the subject of extensive research as to their role in cancer. It has been demonstrated that excessive amounts of HSP are synthesized in tumour tissues, participating in the control processes of cell proliferation, differentiation, and programmed death, as well as contributing to the process of angiogenesis [3, 4]. Overexpression of these proteins can be observed in a number of neoplasms, such as prostate, bladder, breast, ovary, cervical, colon, lung, oesophageal, and kidney cancer [5]. All HSPs are divided into families according to the generally accepted classification depending on their molecular weight expressed in kilodaltons ( $\mathrm{kDa}$ ). Three HSP families play a significant role in neoplastic processes. They are HSP90, HSP70, and HSP27 with molecular weights of 90, 70, and 10-30 KDa (small HSP), respectively.

It has been demonstrated that the expression of HSP27 is a negative prognostic factor in gastric, prostate, and liver cancer, while HSP70 - in bladder, breast, and cervical cancer [6]. HSP90 expression is weakly correlated with breast cancer and is a favourable prognostic factor in endometrial can- 
cer. A marked increase in HSP expression has also been observed under the influence of cytotoxic drugs. This contributes to the survival of neoplastic cells, thus reducing the chances of obtaining response to the applied anticancer treatment [7].

This review highlights the diagnostic and prognostic importance of different classes of heat-shock proteins in oncology. The current state of research on the therapeutic potential of HSPs in cancer treatment is also presented.

\section{The features of the biological activity of HSPs in neoplastic cells}

It has been shown that the structure and properties of HSPs in neoplastic cells remain unchanged compared to normal cells, but neoplastic transformation increases the level of all HSP isoforms and stimulates the expression of their inducible variants [8]. Increased proliferation regarding the neoplastic cells causes relative neoplastic hypoxia, becoming one of the main stressors activating HSP synthesis [9]. HSPs play an important yet ambiguous role in the regulation of apoptosis: HSP10 and HSP60 are pro-apoptotic, while HSP27, HSP70, and HSP9O are anti-apoptotic [2]. Anti-apoptotic proteins prevail in neoplastic cells, and their expression differs depending on the type of cancer; for example, in breast cancer the content of HSP70 increases, and in prostate cancer there is a higher content of HSP90 [10-12].

\section{Apoptosis}

The process of apoptosis can follow diverse molecular pathways. The 2 main apoptotic pathways described in literature are as follows:

- intrinsic - referred to as the mitochondrial pathway due to the main role of this organelle in inducing apoptosis,

- extrinsic - dependent on membrane receptors, which can be affected by external damaging factors.

- Alternative apoptotic pathways are also described in literature:

- pseudoreceptor-induced by granular immune cells (T cells, natural killer cells),

- reticular - triggered by the release of caspases from the endoplasmic reticulum due to an increase in the concentration of calcium ions or the accumulation of abnormal proteins in the cell.

These pathways may intersect or merge into one common pathway that ends in the activation of effector caspases. A number of studies regarding the influence of HSP on the process of apoptosis shed light on its mechanisms and demonstrate that individual chaperones can interact with the apoptotic pathway on several levels [10]. HSPs can modulate apoptosis by temporarily inhibiting the expression of genes encoding the proteins that play an executive role in the process of apoptosis or by attenuating the death signal $[13,14]$.

\section{The role of HSP27 in apoptosis}

Studying HSP27 expression in healthy and neoplastic tissues revealed its close association with various neoplasms. This protein has the ability to inhibit apoptosis by inter- fering with various apoptotic pathways. It has been found that the phosphorylated form of HSP27 can translocate to the nucleus and interact with Daxx (an adaptive protein of the receptor apoptosis activation pathway), preventing its release into the cytoplasm and activation of the death receptor (Fas) [15]. Moreover, the binding of HSP27 to cytochrome $\mathrm{C}$ disrupts apoptosome formation and disturbs the activation of caspase-9 and caspase-3. The HSP27 chaperone can interact directly with procaspase-3, which, in turn, prevents it from being cleaved by caspase-9, and consequently inhibits apoptosis [16].

HSP27 overexpression has been observed in phenotypically angiogenic breast cancer models both in vivo and in vitro. The inhibitory regulation of HSP27 expression causes prolonged remission and decreased expression of angiogenic factors vascular endothelial growth factor (VEGF-A, VEGF-C, and bFGF) [17]. It was also shown that low HSP27 expression correlated with a less aggressive phenotype, while overexpression modulated nemo-like kinase (NLK), which involved in breast cancer apoptosis [18]. In normal cells, NLK is mainly found in the cytoplasm, while in cancer cells it occurs in the nucleus. Inhibition of HSP27 synthesis in breast cancer cells displaced NLK into the cytoplasm, which eventually led to cancer cell apoptosis. This study demonstrates a strong relationship between NLK and HSP27 in the process of apoptosis.

\section{The role of HSP70 in apoptosis}

The anti-apoptotic function of the HSP70 family is well understood. These proteins can act at many different stages of the signalling pathways. In vitro studies (U937 and Wehi-s lines) showed that KN apoptosis is directly dependent on the level of HSP70 synthesis. It has been observed that high expression of HSP70 prevents cell apoptosis induced by actinomycin D, camptothecin, and etoposide. The blocking of apoptosis is accomplished by high-molecular-weight HSPs (including HSP70) binding to caspases, which interferes with the activation of the latter. The neoplastic tissue accumulates a pool of cells with latent mutations, which promotes further tumour development [19].

HSP70 can block apoptosis at the pre-mitochondrial, mitochondrial, and post-mitochondrial levels. This takes place, among others, by interaction with the signalling pathway of the Fas (e.g. DR4 and DR5) and its tumour necrosis factor-related apoptosis-inducing ligand, interfering with the process of changing the mitochondrial membrane permeability and, finally, inhibiting apoptosome formation or protecting nuclear proteins against their cleavage by caspase-3 [20]. The anti-apoptotic effect of HSP70 is observed in cancer cells with either almost unchanged or significantly increased expression of the MYC oncogene. It is well known that the MYC transcription factor ensures a high level of cell proliferation (by increasing the activity of cyclin complexes and cyclin-dependent kinases of the G1 phase of the cell cycle - Cdk4 and (dk2) and also that it can be associated with a high level of apoptosis (by increasing the expression of pro-apoptotic Bcl-2-associated $X$ protein (BAX) protein). It has also been demonstrated that increased MYC expression makes neoplastic 
cells more sensitive to the effect of anti-cancer drugs such as doxorubicin, etoposide, and camptothecin [21]. Under these conditions, increased HSP70 expression inhibits the development of apoptosis induced by these drugs. It appears that HSP70 interference with apoptotic signalling pathways associated with increased MYC expression may be the cause of acquired drug resistance of cancer cells.

\section{The role of HSP90 in apoptosis}

The HSP9O family is one of the most thoroughly studied members of HSPs showing anti-apoptotic properties. The molecular mechanisms of the anti-apoptotic action of HSP9O include a decrease in the activity of caspases- 8 and -3 , a decrease in the number of tumour necrosis factor and Fas receptors, a change in the level of transcription factors p53 and NF- $\mathrm{KB}$, as well as an imbalance of pro- and antiapoptotic proteins from the Bc1-2 family in favour of the latter. The predominance of the expression of anti-apoptotic proteins prevents the reduction of the mitochondrial transmembrane potential.

HSP9O and HSP70 are complementary. Thus, when cytotoxic drugs bind HSP9O there is an increase in HSP70 synthesis, which reflects the activation of the compensatory cellular protective mechanisms. As a consequence, there is an increased probability of survival of cancer cells and resistance to the above-mentioned drugs. Such a phenomenon has been described, for example, in medulloblastoma in children [22]. Blocking of HSP70 and its constitutive isoform, HSC7O (constitutive isoforms HSP), by siRNA causes proteosome-dependent degradation of HSP9O proteins in colon cancer tissues, resulting in extensive tumour apoptosis, as well as intensification of neoplastic cell apoptosis with pharmacological inhibition of HSP90 [11].

\section{Involvement of heat shock proteins in the processes of metastasis}

HSPs have also been studied for their role in the processes of invasion and metastasis, which have a significant impact on overall survival. HSPs were first detected in cells. Subsequently, extracellular HSPS (ex-HSPs), membrane surface HSPs (mHSPs), and ev-HSPs contained in extracellular vesicles (oncosomes and exosomes) were also discovered [23]. Ex-HSPs play a key role in extracellular communication in the neoplastic process, in immune reactions, as well as in other processes, e.g. degenerative processes.

HSPs are released from cells passively, by cell damage or death, or actively by secretion of HSP-containing exosomes. Proteomics showed that oral cancer oncosomes are rich in HSP90, HSP70, HSC70, and HSP105 [23]. It is worth noting that HSP9O $\alpha$ is highly expressed in neoplastic cells and secreted into the extracellular space in the form of free HSP9O $\alpha$ and also as an oncosome load [24].

ex-HSP, ev-HSP, and mHSP can bind to cell surface receptors, stimulate intracellular signalling pathways, be absorbed by endocytosis, or transported molecularly to target cells such as immune cells, cancer cells as well as cancer stem cells, epithelial cells, cancer-associated fibroblasts, mesenchymal stem cells, tumour endothelial cells, and lymphoid endothelial cells [25].
Exosomes derived from primary tumour cells transfer oncogenic factors to cells in the tumour microenvironment and in the pre-metastatic niche [26]. Ex-HSP and ev-HSP play a key role in cancer development by binding to cell surface receptors such as CD91, promoting epithelial-mesenchymal transition (EMT), migration, invasion, heterogeneity, metastasis, drug resistance of cancer cells, and angiogenesis [27-29].

It has been shown, among others, that high serum concentration of soluble HSP27 and phosphorylated HSP27 in colorectal cancer patients who had undergone removal of lung metastases was associated with worse relapse-free and overall survival [30].

\section{Heat shock proteins as biological markers of neoplastic process}

Depending on the type of tumour and its stage of advancement, HSPs show differential expression; therefore, HSP testing may be of prognostic value.

The conducted studies have provided controversial results. The association between HSP expression and prognosis was demonstrated in colorectal cancer, whereas such a correlation was not found in hepatocellular carcinoma, [30, 31]. Decreased HSP27 expression was observed in the early stages of colorectal cancer (grade I and II), while in advanced stages (grade III and IV), increased HSP27 expression was demonstrated [32]. Intracellular HSP27 overexpression is observed in patients with prostate cancer [33]. The prognostic significance of HSP27 overexpression was found in patients with meningioma [34].

Given the ability of HSP to be released into the extracellular space, it has become appropriate to study ex-HSP as a potential cancer biomarker in body fluids using fluid biopsy.

In women with breast cancer, the levels of HSP27 in the serum and tumour microenvironment were significantly higher compared to the control group [35]. A high level of HSP27 was also demonstrated in the interstitial fluid isolated from the primary tumour tissue [36].

HSP70 is often found on the cancer cell plasma membrane and is released into the bloodstream through exosomes. Serum HSP70 levels were significantly higher in patients with lung cancer (both squamous and adenocarcinoma) than in the control group [37]. Serum HSP70 levels correlate significantly with total tumour volume in both types of cancer. The use of HSP70 as a tool for the early diagnosis of cervical cancer was demonstrated in both in vitro and in vivo studies [38]. HSP70 was overexpressed in patients in the first and second stage of the disease. Inhibition of HSP70 expression reduced the mobility of cancer cells and their invasive capacity. Increased expression of HSP70 was also demonstrated in the serum of patients with breast cancer, colorectal cancer, and acute myeloid leukaemia [39]. Moreover, longer survival times were observed in patients with high levels of anti-HSP antibodies.

Promising results were also obtained by studying extracellular and exosomal HSPs as diagnostic and prognostic biomarkers in patients with head and neck squamous cell carcinoma (HNSCC) [23] and prostate cancer [24]. 
HSP-rich exosomes were secreted by HNSCC cells with high metastatic capacity. They contained a significantly higher amount of tumour necrosis factor receptor-associated protein 1 (TRAP1), Hsp90 $\beta$, Hsp90 $\alpha$, Hsp105/HspH1, and Hsp72 compared to HNSCC with low metastatic potential. Patients with tumours demonstrating high levels of TRAP1 or HSP90 $\beta$ are characterised by an unfavourable prognosis. Patients with stage I and II HNSCC showed high expression of TRAP1 and Hsp105, while patients with stage III and IV had increased expression of HSP9O $\alpha / \beta$. Studies on 3-dimensional models of PC-3 castration-resistant prostate cancer cell lines showed a significantly higher secretion of ex-HSP9O $\alpha$ by hypoxic cells compared to smaller tumours and 2-dimensional cultures in which cells are much better oxygenated [24]. In this model, ex-HSP90 $\alpha$ was released in large quantities, while ev-HSP90 $\alpha$ was practically undetectable.

High HSP9O expression was repeatedly demonstrated in breast cancer patients, but this only applies to ductal carcinoma, as in the case of the lobular subtype, a clear decrease in HSP9O expression was found [40]. The study of the heterogeneity of breast cancer showed a correlation of increased expression of several genes responsible for HSP90 synthesis with an increased risk of death and aggressive cancer phenotype. Different HSP9O isoforms are associated with negative prognosis for various neoplastic disease subtypes. It was suggested that increased levels of HSP90 may be a prognostic factor for aggressive neoplasms (HER2/ER2 status) with higher risk of recurrence and distant metastases [41].

Noteworthy is the correlation of the extracellular level of HSP9O with the EMT of neoplastic cells, which is a sign of neoplastic progression [42].

This is confirmed by morphological changes in cancer cells corresponding to the mesenchymal phenotype - for example, elongated shape, loss of cell-cell junctions and the acquisition of the ability of cells to migrate, and thus transformation from tight cubic epithelial cells pattern into loosely dispersed groups. This is accompanied by changes in the expression of EMT markers (E-cadherin, N-cadherin, Zeb1, Zeb2). The decisive role of ex-HSP90-LRP1 (lipoprotein receptor-related protein - LRP1) in the initiation of the migration capacity of prostate cancer cells has been confirmed. Increased levels of ex-HSP9O are associated with the aggressive neoplastic cell phenotype, which prompts research into the possibility of using ex-HSP9O in cancer diagnosis and treatment [43]. The prognostic value of HSP9O in gastric and colorectal cancer has also been confirmed, its high expression indicating positive correlation with invasiveness and metastasis [44]. HSP9O was suggested to be an indicator of KN differentiation in lung adenocarcinoma [45]. Of the 4 isoforms of HSP9O that are present in humans, 2 of them - GRP94 and TRAP1 - show higher expression compared to the others - HSP9O $\alpha$ and HSP90 $\beta$ - in patients with small cell lung cancer. The diversified expression of these isoforms indicates their different participation in carcinogenesis [46]. Increased expression of Hsp90- $\beta$ can be used for the differential diagnosis of pleural effusion in lung cancer and correlates with pathomorphological differentiation, tumour size, and lymph node metastases as well as the aggressive course of the disease [47].

The presented results indicate the purposefulness of research into the role of HSPs in the early diagnosis of cancer and the importance of HSPs as biological markers of cancer progression.

\section{Heat shock proteins as a target in anti-cancer therapy}

Considering the importance of HSPs at various stages of the neoplastic process, they seem to be an interesting therapeutic target. The development of HSP inhibitors is, however, quite a challenge due to the lack of selectivity towards neoplastic and normal cells, and therefore high toxicity, as well as the problem of removing them from the cell by means of membrane transporters responsible for the phenomenon of multidrug resistance (MDR).

Geldanamycin (GA), an antibiotic from the ansamycin family, was the first HSP90 inhibitor. Studies have shown that GA stops tumour proliferation by inhibiting the activity of Src tyrosine kinase and blocking the ATP binding site in HSP9O, leading to proteasomal degradation of these proteins [48]. The structure of GA was then modified and 17-AAG (tanespimycin) was developed. 17-AAG was the first HSP90 inhibitor to be used in human clinical trials. In addition, in vivo studies were carried out to test 17-AAG as a tool to induce apoptosis and inhibit cell proliferation by increasing the concentration of cytochrome c, caspase-9, and caspase-3 [49]. The significant anti-neoplastic effect allowed 17-AAG to enter phase I and II clinical trials in patients with advanced breast cancer, pancreatic cancer, and melanoma, where it was used both as monotherapy and in combination with gemcitabine, cisplatin, bortezomib, and trastuzumab [50-52].

Other GA derivatives such as 17-DMAG, IPI-504 (retaspimycin) and IPI-493 were also tested [53]. Following the success of phase I and II clinical trials, IPI504, as well as other HSP90 inhibitors such as STA-9090 and AUY922, were subjected to phase III studies in the non-small cell lung cancer (NSCLC) population. Their efficacy has not been demonstrated in a random population of NSCLC patients [54-56].

However, the results of studies on another HSP9O inhibitor, dorsomorphin, are encouraging. It was demonstrated that dorsomorphin reduces HSF1 Ser320 phosphorylation and nuclear translocation, as well as the level of nuclear HSF1 in cancer cells, inducing their apoptosis [57]. Moreover, in the conducted in vitro studies, it sensitized cancer cells to HSP9O and proteasome inhibitors and inhibited HSP70 expression induced by these inhibitors. In mice, dorsomorphin enhances neoplastic cell apoptosis and inhibits HSP70 expression and tumour growth.

The phenomenon of multi-drug resistance is a serious limitation of many anti-cancer therapies and is the subject of ongoing research. Eleven HSP9O inhibitors containing an isoxazolonaphthoquinone core have been synthesized and tested in tumour models of small cell lung cancer and MDR colorectal adenocarcinoma [58]. The efficacy was determined on the basis of the inhibition of cell growth, ac- 
tivity, and expression of P-glycoprotein (P-gp) - the membrane transporter responsible for MDR. For some of the tested compounds, interaction with P-gp was observed through inhibition of its ATPase activity and decreased P-gp expression in cancer cells.

In order to obtain selectivity of HSP inhibitors towards neoplastic cells, attempts were made to combine HSP9O inhibitors with anti-cancer drugs or radiotherapy, as well as to deliver compounds to the cancer cells, e.g. in nanosomes (drug delivery systems) [7]. The anti-neoplastic activity of the combination of the HSP90 XL888 inhibitor and vemurafenib was demonstrated in patients with melanoma with the BRAFV600 mutation [59].

It was demonstrated that the new derivative of 5-resorcinol triazolone (PTP-Ganetespib) was active in breast cancer cells, including MDA-MB-231 triple-negative breast cancer, and it was approved for phase III clinical trials [60].

5-aryl-3-thiophene-2-yl-1H-pyrazole, another new HSP90 inhibitor, was tested on MCF7 and HepG2 (hepatocellular carcinoma) cell lines. The compound containing the thiophene group showed the highest antiproliferative and apoptotic activity against HepG2 cells. It caused cell cycle arrest in the $\mathrm{G} 2$ phase, a 7.7-fold increase in caspase-3, inhibition of $\mathrm{Hsp90}(\mathrm{IC50}=2.67 \pm 0.18 \mu \mathrm{M})$ in vitro, and also decreased Hsp90 levels by $70.8 \%$. Moreover, it significantly decreased the level of Hsp90 client proteins (Akt, c-Met, c-Raf, and EGFR) and gave a 1.57-fold increase of Hsp70 [61].

VER155008, a new inhibitor that targets HSP70, blocks the phosphorylation of the PI3K/AKT/mTOR and MEK/ERK signalling pathways. VER155008 may reduce the proliferation of PC12 pheochromocytoma and induce apoptosis, as well as inhibit cell migration and invasion [62]. In addition, VER-155008 inhibits the proliferation of breast and colon cancer lines (HCT116 and BT474) and induces the degradation of HSP90. Induction of caspase-3/7-dependent apoptosis in BT474 cells and caspase-independent death of HCT116 cells was also observed.

2-Phenylethylsulfonamide (PES) is a selective inhibitor of HSP7O function in various types of KN, showing lower toxicity to normal cells. PES was shown to inhibit the proliferation of oral squamous cell carcinoma cell lines in vivo and in vitro, arrest the cell cycle arrest, and trigger apoptosis. PES inhibited the expression of X-linked inhibitor of apoptosis protein (XIAP), c-IAP1, p-AKT, and p-ERK and disrupted the interaction between Hsp70 and XIAP [63].

Recently, it was found that JG-98, an inhibitor containing pyridinic acid modified benzothiazole, showed promising antiproliferative activity in cancer cells. However, pyridinic acid causes undesirable disturbances in biochemical and cellular reactions. Therefore, it has been replaced with neutral pyridine. Pyridine-modified benzothiazoles, such as $J G 2-38$, retain promising antiproliferative effects in breast and prostate cancer cell lines [64].

Cantharidin (CTD), a terpenoid derivative, inhibits HSP70 expression by blocking HSF1 binding to the HSP70 promoter [65]. The introduction of thermosensitive CTD closing liposomes induces cell apoptosis, blocking the response to heat shock and subsequent expression of HSP70 and BAG3 in cervical cancer [66].
Apoptozole (AZ) is an inhibitor of HSP70 that promotes neoplastic cell apoptosis by permeabilising the lysosomal membrane. AZ-mediated disruption of lysosomal function also inhibits protective autophagy and promotes cell apoptosis in multiple cancer cell lines [67].

The assessment of the effect of dihydroartemisinin (DHA) on HSP70 expression in PC-3 prostate cancer cells has demonstrated that DHA inhibits HSP70 expression in PC-3 cells. DHA interferes with the formation of the association between dATP, cytochrome c, Apaf-1, caspase-3, apoptosis-inducing factor, and HSP70, and consequently inhibits the anti-apoptotic capacity of HSP70 and promotes apoptosis of PC-3 cells [68].

The search for inhibitors targeting HSP27 is extremely difficult because HSP27, unlike other heat shock proteins, is independent of ATP [69]. However, a new strategy for inhibiting HSP27 by inducing cross-linking of the HSP27 protein has been suggested. Introducing inserts into the disulphide bond alters the cross-linking of HSP27, which interferes with HSP27 dimerization and function. Moreover, altered HSP27 dimerization may sensitize cancer cells that express HSP27 [70].

Of the HSP27 inhibitors, OGX-427, an antisense oligonucleotide (apatorsen), is the most prominent. Its effectiveness has been assessed in preclinical and clinical trials [71]. High therapeutic activity of OGX-427 was demonstrated in combination with traditional anti-cancer drugs (gemcitabine, docetaxel) [72,73], as well as with other chaperone inhibitors and proteotoxic agents, demonstrating significant efficacy. OGX-427, co-administered with the autophagy inhibitor chloroquine, significantly inhibited the growth of prostate cancer in animal models [74]. Complex administration of the Hsp90 inhibitor PF-04928473 together with OGX-427 revealed effective inhibition of cancer cell growth and induction of apoptosis. In a castration-resistant prostate cancer (CRPC) model, the above combination inhibited tumour growth and prolonged animal survival [75].

Bortezomib significantly inhibited HSP27 expression. Treatment with bortezomib or OGX-427 inhibited proliferation and promoted apoptosis in U266 cell lineage and significantly reduced HSP27 expression. Moreover, Bcl-2 expression was significantly decreased, while BAX expression was increased by bortezomib and OGX-427. There was no significant difference between the bortezomib group and OGX-427 in vitro. HSP27 correlates positively with $\mathrm{Bcl}-2$ expression and negatively with BAX expression in U266 cells. Bortezomib promotes apoptosis in multiple myeloma cells [76]. Recently, research on ivermectin as an inhibitor of HSP27 capable of enhancing oncogene targeting in cancer models has been presented [77].

\section{Conclusions}

Numerous experimental studies confirm the assumption of using HSPs as clinical biomarkers and therapeutic targets in oncology. HSPs have been shown to be overexpressed in some types of cancer and to play an important role in oncogenic processes, and, therefore, they may be of great prognostic value. HSPs are associated with process- 
es typical of cancer development and progression, such as cell proliferation, invasion, and metastasis. In addition, HSPs influence cell survival mechanisms by interacting with key apoptotic proteins. This allows them to be regarded as promising targets for various therapies. The inhibition of HSP with various preparations is the subject of research in a number of cancers. However, more research is needed to refine the efficacy of HSP in combination with other traditional markers for cancer diagnosis and prognosis of their progression.

\section{The authors declare no conflict of interest.}

\section{References}

1. Toth ME, Gombos I, Santha M. Heat shock proteins and their role in human diseases. Acta Biolszeged 2015; 59: 121-141.

2. Milani A, Basirnejad M, Bolhassani A. Heat-shock proteins in diagnosis and treatment: an overview of different biochemical and immunological functions. Immunotherapy 2019; 11: 215-239.

3. Sreedhar AS, Csermely P. Heat shock proteins in the regulation of apoptosis: new strategies in tumor therapy: a comprehensive review. Pharmacol Ther 2004; 101: 227-225.

4. Almeida M, Nascimento JL, Herculano AM, Crespo-López ME. Molecular chaperones: toward new therapeutic tools. Biomed Pharmacother 2011; 65: 239-243.

5. Seigneuric R, Mjahed H, Gobbo J. Heat shock proteins as danger signals for cancer detection. Front Oncol 2011; 10: 37.

6. Wu J, Liu T, Rios Z, Mei Q, Lin X, Cao S. Heat shock proteins and cancer. Trends Pharmacol Sci 2017; 38: 226-256.

7. Shevtsov M, Multhoff G, Mikhaylova E, Shibata A, Guzhova I, Margulis B. Combination of anti-cancer drugs with molecular chaperone inhibitors. Int J Mol Sci 2019; 20: 5284.

8. Ban HS, Han T-S, Hur K, Cho H-S. Epigenetic alterations of heat shock proteins (HSPs) in cancer. Int J Mol Sci 2019; 20: 4758.

9. Semenza GL. The hypoxic tumor microenvironment: a driving force for breast cancer progression. Biochim Biophys Acta 2016; 1863: 382-391.

10. Wang X, Chen M, Zhou J, Zhang X. HSP27, 70 and 90, anti-apoptotic proteins, in clinical cancer therapy (review). Int J Oncol 2014; 45:18-30.

11. Massey AJ, Williamson DS, Browne H, et al. A novel, small molecule inhibitor of $\mathrm{Hsc70/Hsp70} \mathrm{potentiates} \mathrm{Hsp90} \mathrm{inhibitor} \mathrm{induced}$ apoptosis in HCT116 colon carcinoma cells. Cancer Chemother Pharmacol 2010; 66: 535-545.

12. Taha E, Ono K, Eguchi T. Roles of extracellular HSPs as biomarkers in immune surveillance and immune evasion. Int J Mol Sci 2019; 20: 4588.

13. Beere HM, Wolf BB, Cain K, et al. Heat shock protein 70 inhibits apoptosis by preventing recruitment of procaspase- 9 to the Apaf-1 apoptosome. Nat Cell Biol 2000; 2: 469-475.

14. Beere H. Stressed to death: regulation of apoptotic signaling pathways by the heat shock proteins. Sci STKE 2001; 93: RE1.

15. Charette SJ, Lavoie JN, Lambert H, Landry J. Inhibition of daxx-mediated apoptosis by heat shock protein 27. Mol Cell Biol 2000; 20: 7602-7612.

16. Concannon CG, Gorman AM, Samali A. On the role of Hsp27 in regulating apoprosis. Apoptosis 2003; 8: 61-70.

17. Straume O, Shimamurad T, Lampa M, et al. Suppression of heat shock protein 27 induces long-term dormancy in human breast cancer. Proc Natl Acad Sci USA 2012; 109: 8699-8704.

18. Hallgren GS, Masoumi KC, Zarrizi R, et al. Association of nu-clear-localized nemo-like kinase with heat- shock protein 27 inhibits apoptosis in Human Breast Cancer Cells. PLoS One 2014; 9: e96506.

19. Jindal DG, Jindal V, Joshi S, Bhojia I, Chawdhry A. Heat shock proteins in pathology: a review. J Pierre Fauchard Academy (India Section) 2016; 30: 84-87.
20. Stankiewicz A, Livingstone AM, Mohseni N, Mosser D. Regulation of heat-induced apoptosis by Mcl-1 degradation and its inhibitionby Hsp70. Cell Death Differ 2009; 16: 638-647.

21. Rimpi S, Nilsson JA. Metabolic enzymes regulated by the Myc oncogene are possible targets for chemotherapy or chemoprevention. Biochem Soc Trans 2007; 35: 305-310.

22. Alexiou GA, Vartholomatos G, Stefanaki K, et al. Expression of heat shock proteins in medulloblastoma. J Neurosurg Pediatr 2013; 12: 452-457.

23. Ono K, Eguchi T, Sogawa C, et al. HSP-enriched properties of extracellular vesicles involve survival of metastatic oral cancer cells. J Cell Biochem 2018; 119: 7350-7362.

24. Eguchi T, Sogawa C, Okusha Y, et al. Organoids with cancer stem cell-like properties secrete exosomes and HSP9O in a 3D nanoenvironment. PLoS One 2018; 13: e0191109.

25. Fujiwara T, Eguchi T, Sogawa C, et al. Carcinogenic epithelial-mesenchymal transition initiated by oral cancer exosomes is inhibited by anti-EGFR antibody Cetuximab. Oral Oncol 2018; 86: 251-257.

26. Tai YL, Chen KC, Hsieh JT, Shen TL. Exosomes in cancer development and clinical applications. Cancer Sci 2018; 109: 2364-2374.

27. Najafi M, Goradel NH, Farhood B, et al. Tumor microenvironment: Interactions and therapy. J Cell Physiol 2019; 234: 5700-5721.

28. Nagaraju GP, Long TE, Park W, et al. Heat shock protein 90 promotes epithelial to mesenchymal transition, invasion, and migration in colorectal cancer. Mol Carcinog 2015; 54: 1147-1158.

29. Nolan KD, Kaur J, Isaacs JS. Secreted heat shock protein 90 promotes prostate cancer stem cell heterogeneity. Oncotarget 2017; 8: 19323-19341.

30. Schweiger T, Nikolowsky C, Starlinger P, at al: Stromal expression of heat-shock protein 27 is associated with worse clinical outcome in patients with colorectal cancer lung metastases. PLoS One 2015; 10: e0120724.

31. Eto D, Hisaka T, Horiuchi H, et al. Expression of HSP27 in hepatocellular carcinoma. Anticancer Res 2016; 36: 3775-3779.

32. Rizk OK, El-Rashidy M A. HSP27 immunohistochemical expression in colorectal carcinoma: does it have a prognostic role? Egyptian J Pathol 2016; 36: 71-75.

33. Miyake H, Murakami M, Kurahashi T, Yamanaka K, Hara I, Fujisawa M. Enhanced expression of heat shock protein 27 following neoadjuvant hormonal therapy is associated with poor clinical outcome in patients undergoing radical prostatectomy for prostate cancer. Anticancer Res 2006; 26: 1583-1587.

34. Assimakopoulou M. Humanmeningiomas: immunohistochemical localization of progesterone receptor and heat shock protein 27 and absence of estrogen receptor and PS2. Cancer Detect Prev 2000; 24: 163-168.

35. Banerjee S, Lin C, Skinner K, et al. Heat shock protein 27 differentiates tolerogenic macrophages that may support human breast cancer progression. Cancer Res 2011; 71: 318-327.

36. Mischak H, Allmaier G, Apweiler R, et al. Recommendations for biomarker identification and qualification in clinical proteomics. Sci Transl 2010; 2: 46ps42.

37. Gunther S, Ostheimer C, Stangl S, et al. Correlation of Hsp70 serum levels with gross tumor volume and composition of lymphocyte subpopulations in patients with squamous cell and adeno non-small cell lung cancer. Front Immunol 2015; 6: 556.

38. Garg M, Kanojia D, Saini S, et al. Germ cell-specific heat shock protein 70-2 is expressed in cervical carcinoma and is involved in the growth, migration, and invasion of cervical cells. Cancer 2010; 116: 3785-3796.

39. Gunaldi M, Afsar CU, Okuturlar Y, et al. Elevated serum levels of heat shock protein 70 are associated with breast cancer. Tohoku J Exp Med 2015; 236: 97-102.

40.Zagouri F, Sergentanis T, Nonni A, et al. Decreased Hsp90 expression in infiltrative lobular carcinoma: an immunohistochemical study. BMC Cancer 2010; 10: 409.

41. Cheng Q, Chang JT, Geradts J, et al. Amplification and high-level expression of heat shock protein 90 marks aggressive phenotypes of human epidermal growth factor receptor 2 negative breast cancer. Breast Cancer Res 2012; 14: R62.

42. Hance MW, Dole K, Gopal U, et al. Secreted Hsp90 is a novel regulator of the epithelial to mesenchymal transition (EMT) in prostate cancer. J Biol Chem 2012; 287: 37732-37744. 
43. Wong D, Jay D. Emerging roles of extracellular Hsp90 in cancer. Adv Cancer Res 2016; 129: 141-163.

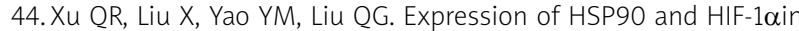
human colorectal cancer tissue and its significance. Asian Pac J Trop Med 2014; 7: 720-724.

45. Wu Y, Huang B, Liu Q, Liu Y. Heat shock protein 90- $\beta$ over-expression is associated with poor survival in stage I lung adenocarcinoma patients. Int J Clin Exp Pathol 2015; 8: 8252-8259.

46. Lee J, Kang K, Kim J, et al. Differential expression of heat shock protein 90 isoforms in small cell lung cancer. Int J Clin Exp Pathol 2015; 8: 9487-9493.

47. Biaoxue R, Min L, Tian F, Wenlong G, Hua L. Elevated Hsp90-beta contributes to differential diagnosis of pleural effusion caused by lung cancer and correlates with malignant biological behavior of lung cancer. BMC Pulm Med 2018; 18: 188.

48. Egorin M, Zuhowski E, Rosen D, Sentz D, Covey J, Eiseman J. Plasma pharmacokinetics and tissue distribution of 17-(allylamino)-17-demethoxygeldanamycin (NSC 330507) inCD2F1 mice1. Cancer Chemother Pharmacol 2001; 47: 291-302.

49. Zhao X, Wang J, Xiao L, et al. Effects of 17-AAG on the cell cycle and apoptosis of $\mathrm{H} 446$ cells and the associated mechanisms. Mol Med Rep 2016; 14: 1067-1074.

50. Schenk E, Hendrickson A, Northfela D, et al. Phase I study of tane-spimycin in combination with bortezomib in patients with advanced solid malignancies. Invest New Drugs 2013; 31: 1251-1256.

51. Pacey S, Gore M, Chao D, et al. Phase II trial of 17-allylamino, 17-demethoxygeldanamycin (17-AAG, tanespimycin) in patients with metastatic melanoma. Invest New Drugs 2012; 30: 341-349.

52. Pedersen K, Kim G, Foster N, Wang-Gillam A, Erlichman C, McWilliams R. Phase II trial of gemcitabine and tanespimycin (17AAG) in metastatic pancreatic cancer: a Mayo Clinic Phase II Consortium study. Invest New Drugs 2015; 33: 963-968.

53. Jhaveri K, Taldone T, Modi S, Chiosis G. Advances in the clinical development of heat shock protein 90 (Hsp90) inhibitors in cancers. Biochim Biophys Acta 2012; 1823: 742-755.

54. Wagner A, Chugh R, Rosen L, at al. A phase I study of the HSP9O in hibitor retaspimycin hydrochloride (IPI-504) in patients with gastrointestinal stromal tumors or soft-tissue sarcomas.Clin Cancer Res 2013; 19: 6020-6029.

55. Kühnel A, Schilling D, Combs S, Haller B, Schwab M, Multhoff G. Radiosensitization of HSF-1 knockdown lung cancer cells by low concentrations of Hsp90 inhibitor NVP-AUY922. Cells 2019 8: 1166.

56. Pillai R, Fennell D, Kovcin V, et al. Randomized phase III study of ganetespib, a heat shock protein 90 inhibitor, with docetaxel versus docetaxel in advanced non-small-cell lung cancer (GALAXY-2). J Clin Oncol 2020; 38: 613-622.

57. Li N, Wang T, Li Z, et al. Dorsomorphin induces cancer cell apoptosis and sensitizes cancer cells to HSP9O and proteasome inhib itors by reducing nuclear heat shock factor 1 levels. Cancer Biol Med 2019; 16: 220-233.

58. Dinić J, Podolski-Renić A, Jovanović M, et al. Novel heat shock protein 90 inhibitors suppress P-glycoprotein activity and overcome multidrug resistance in cancer cells. Int J Mol Sci 2019; 20: 4575.

59. Eroglu Z, Chen Y, Gibney GT, et al. Combined BRAF and HSP90 inhibition in patients with unresectable BRAF (V600E)-mutant melanoma. Clin Cancer Res 2018; 24: 5516-5524.

60. Kang J, Lee J, Tas I, et al. Radiosynthesis, biologica levaluation and preliminary micro PET study of (18)F-labeled 5-resorcinolic triazolone derivative based on ganetespib targeting HSP90. Bioorg Med Chem Lett 2018; 28: 3658-3664.

61. Mohamady S, Ismail M, Mogheith S, Attia Y, Taylor S. Discovery of 5-aryl-3-thiophen-2-yl-1H-pyrazoles as a new class of Hsp90 inhibitors in hepatocellular carcinoma. Bioorganic Chemistry 2020; 94: 103433.

62. Xu F, Lin D, Jiang W, et al. HSP70 inhibitor VER155008 suppresses pheochromocytoma cell and xenograft growth by inhibition of PI3K/AKT/mTOR and MEK/ERK pathways. Int J Clin Exp Pathol 2019; 12: 2585-2594.

63. Jiang L, Xiao J. 2-phenylethynesulfonamide inhibits growth of oral squamous cell carcinoma cells by blocking the function of heat shock protein 70. Biosci Rep 2020; 40: BSR20200079.
64. Shao H, Gestwicki JE. Neutral analogs of the heat shock protein 70 (Hsp70) inhibitor, JG-98. Bioorg Med Chem Lett 2020; 30: 126954.

65. Hsia TC, Lin JH, Hsu SC, et al. Cantharidin induces DNA damage and inhibits DNA repair-associated protein levels in $\mathrm{NCl}-\mathrm{H} 460 \mathrm{hu}$ man lung cancer cells. Environ Toxicol 2015; 30: 1135-1143.

66. Wang S, Xin J, Zhang L, et al. Cantharidin-encapsulated thermalsensitive liposomes coated with gold nanoparticles for enhanced photothermal therapy on A431 cells. Int J Nanomed 2018; 13: 21432160 .

67. Park SH, Baek KH, Shin I, Shin I. Subcellular Hsp70 inhibitors promote cancer cell deathvia different mechanisms. Cell Chem Biol 2018; 25: 1242-1254

68. Kong J, Li S, Ma, Q, Liu L, Zheng LJ. Effects of dihydroartemisinin on HSP70 expression in human prostate cancer PC-3 cells. Andrologia 2019; 51: e13280.

69. Chatterjee S, Burns TF. Targeting heat shock proteins in cancer: a promising therapeutic approach. Int J Mol Sci 2017; 18: 1978.

70. Choi B, Choi SK, Park Y, et al. Sensitization of lung cancer cells by altered dimerization of HSP27. Oncotarget 2017; 8: 105372 105382.

71. Yu E, Ellard S, Hotte S, et al. A randomized phase 2 study of a HSP27 targeting antisense, apatorsen with prednisone versus prednisone alone, in patients with metastatic castration resistant prostate cancer. Investig. NewDrugs 2018; 36: 278-287.

72. Ko A, Murphy P, Peyton J, et al. A randomized, double-blinded, phase II trial of Gemcitabine and Nab-Paclitaxel plus apatorsen or placebo in patients with metastatic pancreatic cancer: the RAINIER Trial. Oncologist 2017; 22: 1427-e129.

73. Rosenberg JE, Hahn NM, Regan MM, et al. Apatorsen plus docetaxel versus docetaxel alone in platinum-resistant metastatic urothelial carcinoma (Borealis-2). Br J Cancer 2018; 118: 1434-1441.

74. Kumano M, Furukawa J, Shiota M, et al. Cotargeting stress-activated Hsp27 and autophagy as a combinatorial strategy to amplify endoplasmic reticular stress in prostate cancer. Mol Cancer Ther 2012; 11: 1661-1671.

75. Lamoureux F, Thomas C, Yin MJ, Fazli L, Zoubeidi A, Gleavem M. Suppression of heat shock protein 27 using OGX-427 induces endoplasmic reticulum stress and potentiates heat shock protein 90 inhibitors to delay castrate-resistant prostate cancer. Eur Urol 2014; 66: 145-155.

76. Li J, Zhang X, Shen J, Guo J, Wang X, Liu J. Bortezomib promotes apoptosis of multiple myeloma cells by regulating HSP27. Mol Med Rep 2019; 20: 2410-2418.

77. Nappi L, Aguda AH, Nakouzi NA, et al. Ivermectin inhibits HSP27 and potentiates efficacy of oncogene targeting in tumor models. Clin Invest 2020; 130: 699-714.

\section{Address for correspondence}

\section{MSc Iryna Boliukh}

Institute of Environmental Engineering and Biotechnology

University of Opole

Opole, Poland

e-mail: iryna.boliukh@uni.opole.pl

Submitted: 2.01 .2021

Accepted: 9.02 .2021 